\title{
Analysis of hydro-turbine non-stationary vibration signals based on CEEMDAN and Wigner-Ville Distribution
}

\author{
Wei Jiang ${ }^{1, \text { a) }}$, Jianzhong Zhou ${ }^{1, \text { b) }}$ \\ ${ }^{1}$ School of Hydropower and Information Engineering, Huazhong University of Science and \\ Technology, \\ Wuhan, 430074, China.

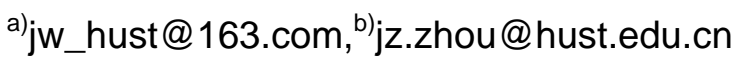

Keywords: Hydro-turbine, Vibration signals, CEEMDAN, Wigner-Ville distribution

\begin{abstract}
This paper proposes a time-frequency method based on complete ensemble empirical mode decomposition with adaptive noise (CEEMDAN) and Winger-Ville distribution (WVD) for hydro-turbine non-stationary vibration signals analysis. Unlike the traditional EMD and EEMD, CEEMDAN can efficiently reduce the mode mixing and has a better convergence. Base on a series of intrinsic mode functions (IMFs) obtained by using CEEMDAN, WVD can effectively suppress the interference of cross-terms and accurately depict the time-frequency characteristics of original vibration signals. The proposed method can be employed to analyze the hydro-turbine non-stationary vibration signals successfully and shows excellent performance.
\end{abstract}

\section{INTRODUCTION}

The vibration signals of hydro-turbine are important indicators for measuring the stability and evaluating the health conditions of the running unit [1]. Generally speaking, vibration signals in engineering practice contain abundant operation information of unit. Due to the influence of various factors, vibration signals are usually non-stationary and fluctuate greatly [2]. Therefore, an effective and practical method of non-stationary vibration signals analysis is significant for the security operation of hydro-turbine.

Recently, with the rapid advance in signal processing technologies, various approaches have been applied in the field of non-stationary signals analysis such as short-time Fourier transform (STFT), wavelet transform (WT) and blind source separation (BSS), and so on [3,4,5]. Empirical mode decomposition (EMD), which is based on the local characteristic time-scale of the data, can be used in nonlinear and non-stationary processes and has been widely applied in many fields successfully [6]. However, there are some inherent limitations in EMD, including the mode mixing, end effects and intrinsic mode function (IMF) criteria. In addition, Wigner-Ville distribution (WVD), which is a kind of bilinear time-frequency distribution, can accurately identify the time-frequency structure of non-stationary signal [7]. But for multi-component signal, the analysis performance of WVD is greatly suffered by the interference of cross-terms.

In order to solve these problems, an time-frequency method based on complete ensemble empirical mode decomposition with adaptive noise (CEEMDAN) and Winger-Ville distribution (WVD) for hydro-turbine non-stationary vibration signals analysis is proposed in this paper (readers are suggested to find more detailed information regarding the CEEMDAN and WVD from $[8,9]$ ) . In the proposed CEEMDAN-WVD, a series of intrinsic mode functions (IMFs) are obtained by applying CEEMDAN into original vibration signals. Compared with those by traditional EMD and EEMD, the obtained IMFs efficiently avoid the mode mixing and have a better convergence because of adding the adaptive noise. Based on the IMFs, WVD can adequately suppress the interference of cross-terms and accurately present the time-frequency characteristics of vibration signals. A real vibration signal of hydro-turbine at the upper guide bearing in a start-up process is employed to demonstrate the effectiveness of the proposed method. Experimental results show that this method can accurately analyze the non-stationary vibration signals and has better performance in comparison of other methods. 


\section{ORGANIZATION OF THE TEXT}

\section{The framework of the proposed CEEMDAN-WVD method}

The flow chart of the proposed CEEMDAN-WVD method is shown in Fig. 1. It provides an effective approach to obtain the accurate time-frequency (TF) distribution of original vibration signal. The detailed steps are stated as follows:

Step 1: The original vibration signal is decomposed by using CEEMDAN. Thus, a series of IMFs, i.e., $I M F_{1}, I M F_{2}, \ldots, I M F_{N}$, can be acquired.

Step 2: Each IMF is analyzed by WVD respectively to obtain the TF distribution of each IMF.

Step 3: The TF distribution of original vibration signal can be acquired by the superposition of the TF distributions of IMFs that are calculated by step 2.

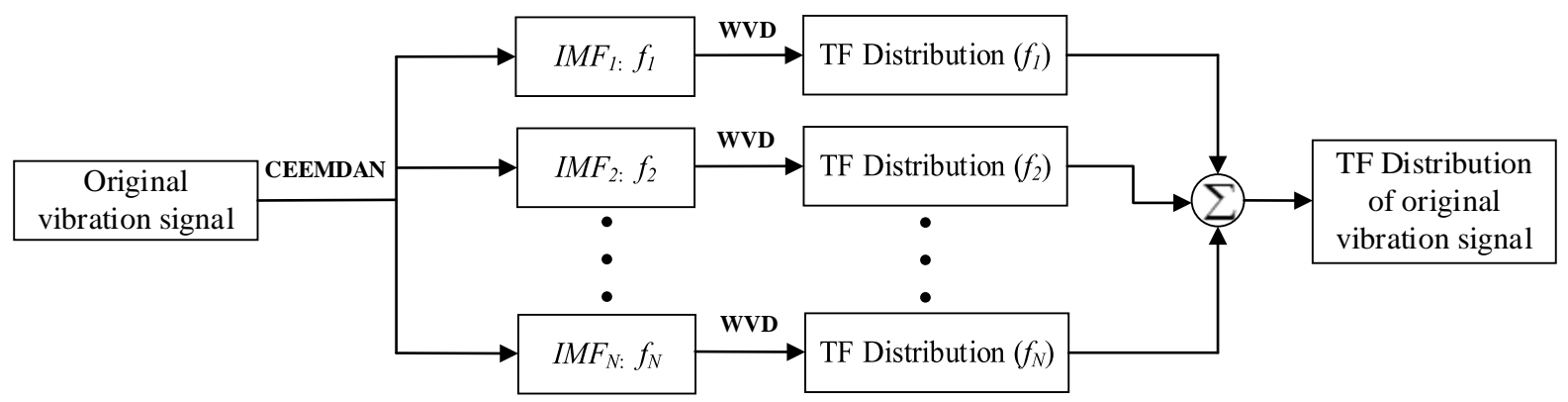

FIGURE 1. Flow chart of the CEEMDAN-WVD method

\section{Experiments and results}

In this section, the proposed CEEMDAN-WVD method is used to analyze the vibration signal of hydraulic turbine, and the obtained result is compared with the result by WVD to validate the effectiveness of CEEMDAN-WVD.

The experimental sample is the main shaft vibration signal of hydro-turbine at the upper guide bearing in a start-up process, which is collected from the 1\# hydroelectric generating unit in Ertan Hydropower Station. The rated speed of unit is $130 \mathrm{rpm}$, the start-up time is 70 seconds and the sampling frequency of the test is $1600 \mathrm{~Hz}$. Fig. 2 shows the FFT spectrum of vibration signal at the upper guide bearing and the starting speed of hydro-turbine. It can be seen from the figure that the distribution of frequency components is more concentrated within a range of 0 to $2.1 \mathrm{~Hz}$, and the entire start-up process lasts about 70 seconds. Because of the influence of some complicated jamming signals, it is difficult for traditional analysis methods to characterize the variation of frequency with the elapse of time accurately. Therefore, due to the advantages of the proposed method for complex non-stationary signal, the sample can be processed by CEEMDAN-WVD to obtain the accurate time-frequency distribution of original signal.

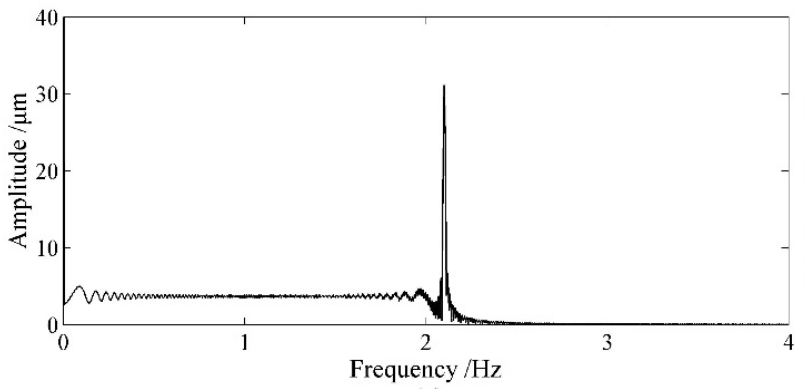

(a)

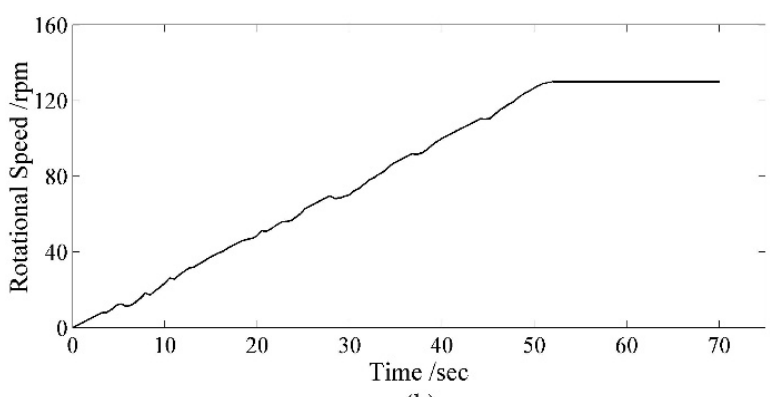

(b)

FIGURE 2. (a) FFT spectrum of hydro-turbine vibration signal (b) Starting speed

Based on the detailed steps of the proposed CEEMDAN-WVD method described in section 2, the time-frequency distribution of original vibration signal can be obtained shown in Fig. 3(a). It is 
obvious that the frequency trajectories are well tracked without any interference terms. The result clearly reflects the varying trend of frequency with time going in the process of unit start-up. Besides, in order to compare with the proposed method, traditional WVD is utilized to analyze the original vibration signal. Fig. 3(b) depicts the time-frequency representation of original signal by WVD. In Fig. 3(b), the WVD has good frequency response accuracy due to its high time-frequency resolution. However, the cross-terms, which is the root cause for spurious frequency components, leads to misinterpretation of the original signal. Thus, the CEEMDAN-WVD method is appropriate for time-frequency analysis with accurate distribution and high energy concentration, especially for the non-stationary vibration signal with cross-terms.

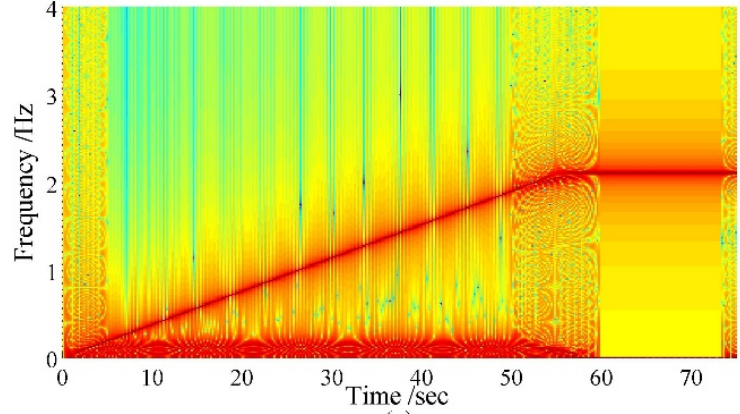

(a)
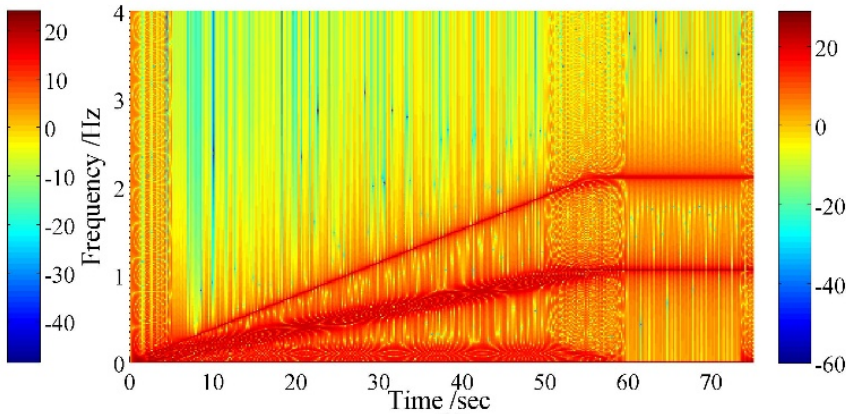

(b)

FIGURE 3. Time-Frequency distribution of hydro-turbine vibration signal (a) CEEMDAN-WVD (b) WVD

\section{CONCLUSION}

EMD and WVD are two widely applied approaches in the field of non-stationary vibration signals processing. In order to further reduce the mode mixing in EMD and suppress the interference of cross-terms in WVD, a novel combination analysis method based on CEEMDAN and WVD is proposed in this paper. Through this method, the high resolution of WVD is remained and the influence of cross-terms can be eliminated effectively. The collected main shaft vibration signal of hydro-turbine at the upper guide bearing in the process of unit start-up is investigated to demonstrate the effectiveness of the proposed method. Experimental results show that the CEEMDAN-WVD method can effectively handle the non-stationary vibration signal and then obtain the accurate time-frequency distribution without spurious frequency components. Furthermore, the proposed method is compared with a time-frequency method based on WVD. From the performance comparison between different methods, the CEEMDAN-WVD presents the real time-frequency distribution of original vibration signal without the effect of cross-terms. The proposed CEEMDAN-WVD method is suitable for the non-stationary vibration signal processing due to its accurate time-frequency analysis result and high energy concentration, and it shows great potential in condition monitoring and fault detection for other mechanical equipment.

\section{ACKNOWLEDGMENTS}

This work was financially supported by the National Nature Science Foundation of China (No.51239004, 51579107).

Corresponding Author: Jianzhong Zhou. Tel.: +86 02787543127. E-mail address: jz.zhou@hust.edu.cn

\section{REFERENCES}

[1] X. An, J. Yang, Denoising of hydropower unit vibration signal based on variational mode decomposition and approximate entropy, T I MEAS CONTROL, 38 (2016) 282-292.

[2] W. Su, X. Li, C. Lan, S. An, J. Wang, F. Li, Chaotic dynamic characteristics of pressure 
fluctuation signals in hydro-turbine, J MECH SCI TECHNOL, 30 (2016) 5009-5017.

[3] M.K. Kıymık, İ. Güler, A. Dizibüyük, M. Akın, Comparison of STFT and wavelet transform methods in determining epileptic seizure activity in EEG signals for real-time application, COMPUT BIOL MED, 35 (2005) 603-616.

[4] Y. Wang, Z. He, Y. Zi, Enhancement of signal denoising and multiple fault signatures detecting in rotating machinery using dual-tree complex wavelet transform, MECH SYST SIGNAL PR, 24 (2010) 119-137.

[5] A.K. Takahata, E.Z. Nadalin, R. Ferrari, L.T. Duarte, R. Suyama, R.R. Lopes, J.M.T. Romano, M. Tygel, Unsupervised Processing of Geophysical Signals: A Review of Some Key Aspects of Blind Deconvolution and Blind Source Separation, IEEE SIGNAL PROC MAG, 29 (2012) 27-35.

[6] D. Wenliao, G. Zhiqiang, G. Xiaoyun, X. Guizhong, W. Liangwen, W. Zhiyang, T. Jianfeng, L. Chengliang, Multifractal characterization of mechanical vibration signals through improved empirical mode decomposition-based detrended fluctuation analysis, Proceedings of the Institution of Mechanical Engineers, Part C: Journal of Mechanical Engineering Science, (2016).

[7] C. Xu, C. Wang, W. Liu, Nonstationary Vibration Signal Analysis Using Wavelet-Based Time - Frequency Filter and Wigner - Ville Distribution, Journal of Vibration and Acoustics, 138 (2016) (051009-1)-(051009-9).

[8] Y. Lei, Z. Liu, J. Ouazri, J. Lin, A fault diagnosis method of rolling element bearings based on CEEMDAN, Proceedings of the Institution of Mechanical Engineers, Part C: Journal of Mechanical Engineering Science, (2015).

[9] G. Andria, M. Savino, A. Trotta, Application of Wigner-Ville distribution to measurements on transient signals, IEEE TRANS. ON INS. AND MEA., 43 (1994) 187-193. 